\title{
Efeitos da suplementação de ômega 3, magnésio e vitamina B12 na função cognitiva e estado nutricional em idosos com Alzheimer
}

\author{
Effects of the supplementation of omega 3, magnesium and vitamin B12 in the cognitive \\ function and nutritional status in elderly with Alzheimer
}
Efectos de suplementación de ômega 3, magnesio y vitamina B12 en la función cognitiva y estado nutricional en idosos con Alzheimer

Ana Paula Gomes da Cunha ${ }^{1 *}$, Layane dos Santos Solano¹, Odara Maria de Sousa Sá1.

\section{RESUMO}

Objetivo: Avaliar os efeitos da suplementação na função cognitiva e estado nutricional em idosos com Alzheimer de instituição filantrópica. Métodos: Estudo de casos múltiplos, aprovado pelo comitê de ética e pesquisa da CONEP/Plataforma Brasil (N².423.431). Foi realizado diagnóstico nutricional: peso, altura, IMC, circunferência da cintura e panturrilha e a avaliação da função cognitiva pelo mini exame mental; após iniciouse a suplementação de ômega 3 (2 cápsulas sendo 1000mg de DHA), magnésio (1 cápsula de $400 \mathrm{mg}$ ) e

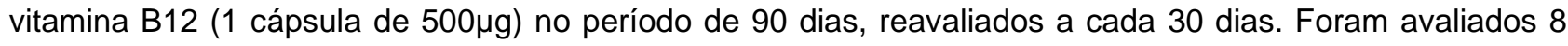
idosos, divididos em 02 grupos: Grupo Controle ( $\mathrm{N}=4$, não suplementados) e Grupo Suplementado $(\mathrm{N}=4)$.Resultados: Os idosos com Alzheimer apresentavam sobrepeso e risco elevado DCV e baixa função cognitiva no período inicial, posteriormente observou-se diferença significativa no aumento do peso após 60 dias quando comparado com período inicial $(p$-valor $\leq 0,05)$ e na circunferência da cintura. $O$ grupo suplementado aumentou sua pontuação total de $55,8 \%$, enquanto grupo controle reduziu para $25,3 \%$ (p-valor $\leq 0,05$ ). Conclusão: A Suplementação em idosos com Alzheimer ocasionou melhoria significativa na função cognitiva, quanto ao estado nutricional houve alterações relevantes no peso e circunferência da cintura, após 60 dias.

Palavras-chave: Cognição. Ácido alfa-linolênico, Vitamina B12, Difosfato de Adenosina.

\begin{abstract}
Objective: To evaluate the effects of supplementation on cognitive function and nutritional status in elderly people with Alzheimer's disease from a philanthropic institution. Methods: Multiple case study, approved by the ethics and research committee of CONEP / Plataforma Brasil $\left(N^{\circ} 2,423,431\right)$. A nutritional diagnosis was performed: weight, height, BMI, waist circumference and calf and the evaluation of cognitive function by mini mental examination; After starting the omega 3 supplementation (2 capsules being $1000 \mathrm{mg}$ of DHA),

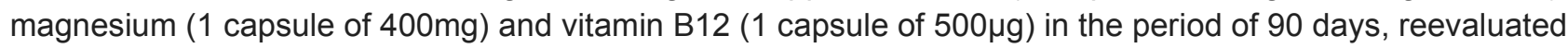
every 30 days. Eight elderly patients were divided into two groups: Control Group ( $N=4$, not supplemented) and Supplemented Group $(\mathrm{N}=4)$. Results: Elderly patients with Alzheimer's disease were overweight and had a high CVD risk and low cognitive function in the initial period there was a significant difference in weight gain after 60 days when compared to baseline ( $p$-value $\leq 0.05$ ) and waist circumference. The supplemented group increased their total score of $55.8 \%$, while control group reduced to $25.3 \%$ ( $p$-value $\leq 0.05$ ). Conclusion: Supplementation in the elderly with Alzheimer's caused a significant improvement in cognitive function; in the nutritional state there were significant changes in weight and waist circumference after 60 days.
\end{abstract}

Keywords: Cognition, Alpha-linolenic acid, Vitamin B12, Adenosine diphosphate.

${ }^{1}$ Centro Universitário Santo Agostinho (UNIFSA),Teresina-PI. *E-mail: anapaullagdc@gmail.com 


\section{RESUMEN}

Objetivo: Evaluar los efectos de la suplementación en la función cognitiva y estado nutricional en ancianos con Alzheimer de institución filantrópica. Métodos: Estudio de casos múltiples, aprobado por el comité de ética e investigación de la CONEP / Plataforma Brasil ( $N^{\circ}$ 2.423.431). Se realizó diagnóstico nutricional: peso, altura, IMC, circunferencia de la cintura y pantorrilla y la evaluación de la función cognitiva por el mini examen mental; Después de iniciarse la suplementación de omega 3 (2 cápsulas siendo 1000 mg de DHA), magnesio

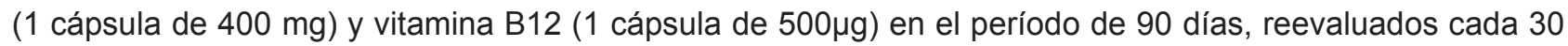
días. Se evaluaron 8 ancianos, divididos en 2 grupos: Grupo Control ( $N=4$, no suplementados) y Grupo Suplementado $(\mathrm{N}=4)$. Resultados: Los ancianos con Alzheimer presentaban sobrepeso y riesgo elevado DCV y baja función cognitiva en el período inicial, posteriormente se observó una diferencia significativa en el aumento del peso después de 60 días cuando comparado con el período inicial ( $p$-valor $\leq 0,05)$ y en la circunferencia de la cintura. El grupo suplementado aumentó su puntuación total del 55,8\%, mientras que el grupo control se redujo al 25,3\% ( $p$-valor $\leq 0,05$ ). Conclusión: La Suplementación en ancianos con Alzheimer ocasionó una mejora significativa en la función cognitiva, en cuanto al estado nutricional hubo alteraciones relevantes en el peso y circunferencia de la cintura, después de 60 días.

Descriptores: Cognición. Ácido alfa-linolénico, Vitamina B12, Difosfato de Adenosina.

\section{INTRODUÇÃO}

Segundo o Instituto Brasileiro de Geografia e Estatística (2002) o número de idosos irá triplicar até o ano de 2050 e com isso o aumento do número de doenças degenerativas ligadas a "demência". Dentre elas citase a Doença de Alzheimer (DA) que corresponde de 50 a $60 \%$ dos casos que e consiste em transtorno neurodegenerativo progressivo e fatal que se manifesta por deterioração cognitiva e da memória, comprometendo progressivamente as atividades diárias e aumento dos sintomas neuropsiquiátricos e de alterações comportamentais (ALZHEIMER'S DISEASE INTERNATIONAL, 2016).

A DA possui três estágios evolutivos. No estágio inicial ou leve surgem as primeiras demonstrações da doença como exemplo as dificuldades em lembrar nomes de pessoas. No estágio intermediário ou moderado, é bem comum à alteração no humor, a frustração, o medo. E no estágio final grave, os movimentos são mais complicados, devido déficit na coordenação motora e a memória é ainda mais agravada (CORREIA A, et al., 2015).

A nutrição apresenta impacto sistêmico no organismo, ou seja, alimentação adequada de acordo com a individualidade bioquímica pode auxiliar na prevenção e/ou tratamento de diversas patologias, dentre elas o Alzheimer. Atualmente a literatura científica não apresenta dieta especifica para a DA. Nessa perspectiva, a utilização de nutrientes específicos pode exercer efeito na DA, como o ômega 3, magnésio e vitamina B12. Por apresentar ação anti-inflamatória, e auxiliar na melhora dos sintomas, como depressão, distúrbios no comportamento, hiperatividade e no déficit de atenção; o ômega 3 é importante no processo cognitivo estando envolvido na estrutura e frações das células lipídicas cerebrais (CANSEV M, et al., 2008).

Como outras funções: apresentam ação antioxidante e são essenciais no processo de envelhecimento cerebral, contribuindo para proteção dos neurônios e evitando assim a morte celular (MOLFINO A, et al., 2014; VEDIN I, et al., 2012).

O magnésio apresenta funcionalidade na condução de impulsos nervosos bem como do ritmo cardíaco normal (CANHADA SL, 2015).

É o principal cátion intracelular com concentração livre no citosol de cerca de 0,5 mmol/L. É essencial em todos os tipos de tecidos de mamíferos, para ampla variedade de funções fisiológicas, atuando em todas as etapas de transcrição e tradução protéica e na transdução de sinais intracelulares, também é responsável pela condução de impulsos nervosos bem como do ritmo cardíaco normal (COMINETTI; ROGERO; HORST, 2017; SCHIEFERDECKER; THIEME; HAUSCHILD, 2016). 
A vitamina B12 (cobalamina) atua em funções significativas no organismo em conjunto com o folato que faz parte do grupo das vitaminas do complexo B, no processo do metabolismo celular. Suas principais fontes são produtos lácteos, carne, fígado, peixe e ovos, que adquirem vitamina indiretamente das bactérias. (GREEN R, 2014; ALLEN LH, 2009; COZZOLINO SMF, 2009).

A deficiência da B12 é mais comum em idosos (acometendo cerca de 20\%) devido uma incapacidade de absorção no trato gastrointestinal, ocasionando aumento de homocisteína contribuindo para o desenvolvimento de doenças ateromatosas, também pode resultar em sintomas graves de depressão, comportamentos suicidas, psicose, declínio cognitivo, demência, DA, este último por ela atuar como um cofator na formação de mielina e na fisiologia normal do sistema nervoso. Vale ressaltar que o fígado é o principal local de armazenamento dessa vitamina. (SCHIEFERDECKER; THIEME; HAUSCHILD, 2016; VOGIATZOGLOU A, et al., 2008; COZZOLINO SMF, 2009).

Nesta perspectiva a pesquisa teve como objetivo avaliar os efeitos da suplementação de ômega 3 (Ácido docosaexaenoico - DHA), magnésio e vitamina B12 na função cognitiva e estado nutricional de idosos com doenças de Alzheimer de instituição filantrópica de Teresina - PI.

\section{MÉTODOS}

Esta pesquisa foi aprovada pelo Comitê de Ética e Pesquisa (CEP)/Plataforma Brasil do Centro Universitário Santo Agostinho ( $N^{\circ}$ 2.423.431). Estudos de casos múltiplos, descritivo, quantitativo, explicativo realizado em instituições filantrópicas de Teresina - Pl. Avaliou-se 8 idosos com Alzheimer, divididos em 02 grupos: Grupo Controle ( $\mathrm{N}=4$, não suplementados) e Grupo Suplementado ( $\mathrm{N}=4$, suplementados com ômega 3 , magnésio e vitamina B12) no período de 3 meses (maio a julho de 2018). Os critérios de inclusão utilizados foram: diagnostico clinico de doença Alzheimer, idosos apresentarem boa dicção e boa deglutição e os critérios de exclusão: Idosos em estágio terminal do Alzheimer e idosos com afasia.

O Diagnóstico nutricional foi realizado por meio de parâmetros antropométricos: peso, altura e o cálculo do Índice de Massa Corporal (Peso Atual/ Altura²) e classificação de Lipschitz,(1994); Circunferência do braço (CB) obtida entre o ponto médio do processo acromial da escapula e olecrano da ulna utilizando a referência de Frisancho (1990), Burr e Phillips (1984) e Blackburn e Thornton (1979); Circunferência Muscular do Braço $(\mathrm{CMB})$ foi obtida pela fórmula $\mathrm{CMB}(\mathrm{cm})=\mathrm{CB}(\mathrm{cm})-[0,314 \times \mathrm{DCT}]$ e classificação de Blackburn e Thornton (1979); Circunferência da cintura (CC) aferida entre o último arco costal e a crista ilíaca e utilizando como referência a OMS 2000; Circunferência da panturrilha (CP) realizada no ponto medial da perna em seu maior perímetro onde o idoso estava sentado e com o tornozelo na posição anatômica e o pé com ou sem apoio e classificação de Guigoz (1999) e dobra cutânea tricipital (DCT) foi obtida na face posterior do braço direito no ponto que compreende a distância média entre o acrômio e o processo olecrano da ulna do idoso descrita em percentil e classificada de acordo com Burr e Phillips (1984).

A função cognitiva foi mensurada pelo mini exame mental (Mini Mental State Examination - MMSE), os itens avaliados foram: orientação, retenção, atenção e cálculo, evocação, linguagem e habilidade construtiva, gerando pontuação em cada item avaliado, o resultado final, somatório de todos os itens deveria chegar até 30 pontos sendo esse valor classificado de acordo com o grau de escolaridade do idoso. Foi coletado as medicações fornecidas para os idosos, porém não era objetivo do trabalho avaliar droga-nutriente bem como fazer o controle da dieta através do cardápio padrão. Após diagnostico do estado nutricional e cognitivo foi prescrito e fornecido diariamente ao grupo suplementado os nutrientes ômega 3 (2 cápsulas sendo $500 \mathrm{mg}$ de DHA cada) após o café da manhã e o jantar, magnésio (1 cápsula de $400 \mathrm{mg}$ ) após o lanche da manhã e vitamina B12 (1 cápsula de $500 \mu \mathrm{g}$ ) após o almoço, no período de 90 dias e reavaliados a cada 30 dias através dos seguintes parâmetros: estado nutricional (questionário avaliativo) e função cognitiva (MMSE).

Vale ressaltar que as dosagens admitidas eram de quantidades máximas permitidas pela DRIs e quanto a ingestão de nutrientes os questionários foram aplicados apenas para observarse as dietas dos abrigos eram semelhantes, já que não era intenção dos autores calcular quantitativamente dieta e adequar juntamente com a suplementação, mas sim o foco da suplementação na melhoria cognitiva quanto a patologia Alzheimer. 
Os dados foram processados no Excel 2010, com a análise estatística conduzida no Software R e o SPSS 20.0. Primeiramente foi feita uma análise descritiva das variáveis e foram aplicados testes inferenciais Wilcoxon e Mann whitney nas amostras pareadas, adotando nível de significância de $p \leq 0,005$.

\section{RESULTADOS}

Observou-se nesta pesquisa que em relação ao sexo houve predominância do sexo feminino $62,5 \%$ ( $n=$ 5 , média de idade 85 anos) e $37,5 \%$ do sexo masculino ( $n=3$, média de idade 88 anos), média de idade $87 \pm$ 8,31 anos, mínimo 72 anos e máximo 97 anos.

Em relação à avaliação do estado nutricional quanto aos parâmetros antropométricos avaliados (Tabela 1) houve diferença significativa no peso dos idosos após 60 dias tendo ele aumentado significativamente ( $p$ valor $\leq 0,05)$ quando comparado com período inicial. Além disso também aumentou significativamente a circunferência da cintura, sendo justificado devido ao aumento de massa corporal total dos idosos.

Tabela 1 - Parâmetros antropométricos de idosos com Alzheimer em Teresina, PI.

\begin{tabular}{|c|c|c|c|c|}
\hline Parâmetro & $\begin{array}{c}\text { Diagnostico Nutricional } \\
\text { D } 0\end{array}$ & $\begin{array}{l}\text { Reavaliação } \\
\text { D } 30\end{array}$ & $\begin{array}{l}\text { Reavaliação } \\
\text { D } 60\end{array}$ & $\begin{array}{l}\text { Reavaliação } \\
\text { D } 90\end{array}$ \\
\hline Peso (Kg) & $59,75 \pm 17,85$ & $59,88 \pm 18,06$ & $62,75 \pm 18,34^{\star *}$ & $61,25 \pm 16,82$ \\
\hline $\operatorname{IMC}\left(\mathrm{Kg} / \mathrm{m}^{2}\right)$ & $27,25 \pm 5,82$ & $27,13 \pm 5,74$ & $28,63 \pm 5,93$ & $28,50 \pm 5,78$ \\
\hline $\mathrm{CC}(\mathrm{cm})$ & $95,13 \pm 16,49$ & $96,88 \pm 17,26$ & $99,25 \pm 17,28^{\star *}$ & $98,63 \pm 16,72$ \\
\hline $\mathrm{DCT}(\mathrm{mm})$ & $13,75 \pm 5,52$ & $14,0 \pm 6,00$ & $15,00 \pm 5,68$ & $12,63 \pm 5,63$ \\
\hline Altura & $1,38 \pm 0,52$ & $1,38 \pm 0,52$ & $1,38 \quad 0,52$ & $1,38 \pm 0,52$ \\
\hline CB & $30,13 \pm 4,89$ & $30,25 \pm 4,59$ & $30,25 \pm 4,65$ & $30,38 \pm 4,81$ \\
\hline CMB & $25,63 \pm 4,69$ & $25,88_{ \pm} 4,02$ & $25,50 \pm 3,55$ & $26,13 \pm 4,64$ \\
\hline $\mathrm{CP}$ & $32,88 \pm 4,02$ & $33,25+4,68$ & $33,00+5,11$ & $32,88+5,11$ \\
\hline
\end{tabular}

Fonte: Dados da Pesquisa, 2018.

* Médias e Desvio padrão

** 0,048 e 0,05 diferença estatisticamente significativa a um nível de significância de $5 \%(p \leq 0,05)$.

Legenda: Índice de massa corpórea (IMC), Circunferência da cintura (CC), dobra cutânea tricipital (DCT), Circunferência do braço (CB), Circunferência Muscular do Braço (CMB), Circunferência da panturrilha (CP)

Em relação ao IMC observou-se que as classificações se mantiveram as mesmas na fase inicial e após 90 dias de suplementação sendo os maiores valores de sobrepesos (62,5\%) e desnutridos (25\%) respectivamente como mostra o gráfico 1.

Gráfico 1 - Classificação do IMC dos idosos com Alzheimer, o período inicial e 90 dias após, Teresina, PI, 2018.

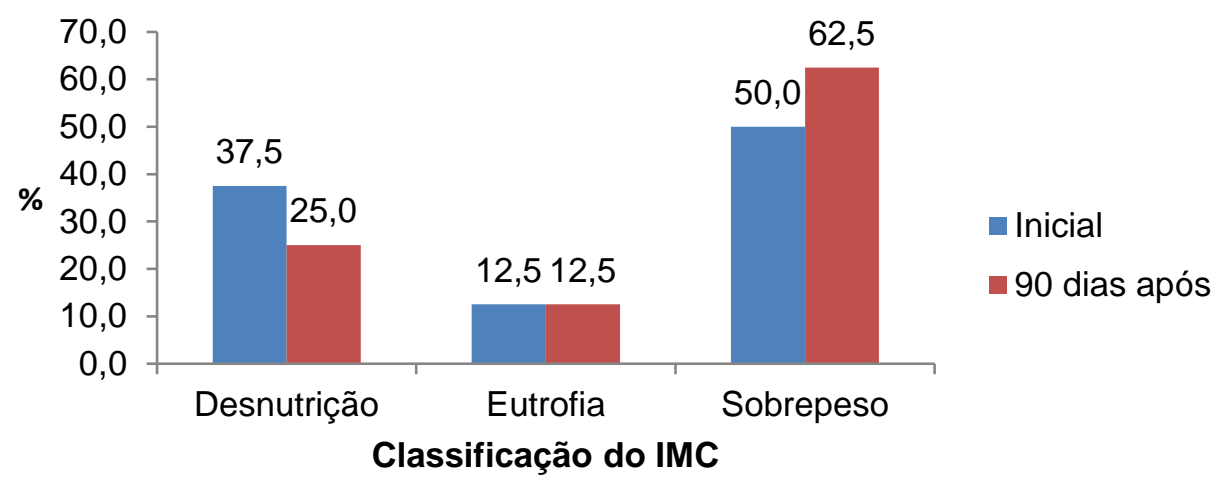

Fonte: Dados da Pesquisa, 2018. 
Quanto à circunferência da cintura observou-se que $62,5 \%$ dos idosos apresentavam risco muito elevado de doenças cardiovasculares (DC) e 37,5\% sem risco DC e 0\% com risco elevado. Porém após o prazo determinado de suplementação o percentual de risco muito elevado para doenças cardiovasculares mantevese os mesmos, enquanto que os sem risco diminuíram para $25 \%$ e os riscos elevados subiram para $12,5 \%$ como consta no Gráfico 2.

Gráfico 2 - Classificação da circunferência da cintura dos idosos com Alzheimer, o período inicial e 90 dias após, Teresina, PI, 2018.

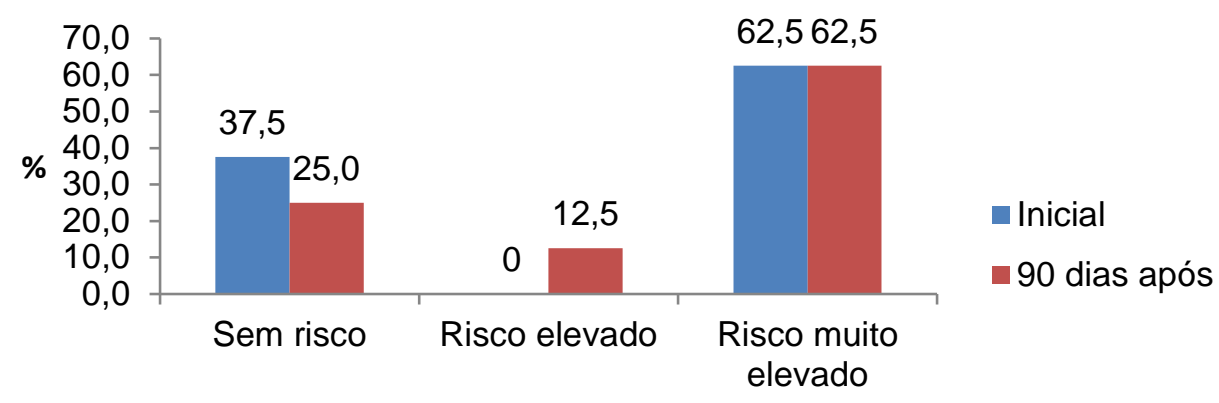

Classificação da circunferência da cintura

Fonte: Dados da Pesquisa, 2018.

Quanto à circunferência muscular do braço $75 \%$ encontraram-se eutróficos e 12,5\% mantiveram-se os mesmos para depleção moderada e leve. Após 90 dias observou-se que os valores para eutrofia e depleção leve foram $37,5 \%$ e depleção moderada subiu para $25 \%$ dados esses que podem ser verificados no Gráfico 3.

Gráfico 3 - Classificação da circunferência muscular do braço dos idosos com Alzheimer, o período inicial e 90 dias após, Teresina, PI, 2018.

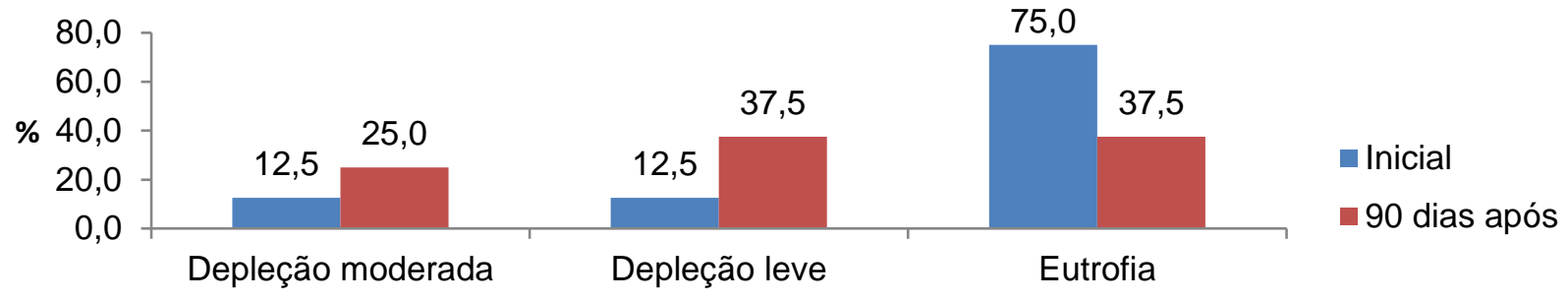

Circunferência muscular do braço

Fonte: Dados da Pesquisa, 2018.

Tabela 2 - Avaliação do MMES de idosos com Alzheimer em Teresina durante 90 dias

\begin{tabular}{lcccc}
\hline Parâmetro & $\begin{array}{c}\text { Diagnostico } \\
\text { Nutricional } \\
\text { D0 }\end{array}$ & $\begin{array}{c}\text { Reavaliação } \\
\text { D30 }\end{array}$ & $\begin{array}{c}\text { Reavaliação } \\
\text { D60 }\end{array}$ & $\begin{array}{c}\text { Reavaliação } \\
\text { D90 }\end{array}$ \\
\hline Orientação & $3,88_{ \pm} 3,52$ & $6,13 \pm 3,04$ & $6,38 \pm 2,92^{* *}$ & $6,75 \pm 2,82^{* *}$ \\
Retenção & $2,38 \pm 0,92$ & $2,50 \pm 0,76$ & $2,13 \pm 0,99$ & $2,25_{ \pm} 1,04$ \\
Atenção Cálculo & $1,13 \pm 1,36$ & $0,13 \pm 0,35$ & $0,25 \pm 0,46$ & $0,13 \pm 0,35$ \\
Evocação & $1,75 \pm 1,28$ & $2,38 \pm 1,06$ & $2,25 \pm 1,16$ & $2,25 \pm 1,16$ \\
Linguagem & $6,25 \pm 1,49$ & $6,75 \pm 0,89$ & $5,88_{ \pm} 2,23$ & $5,75 \pm 2,12$ \\
Habilidade Construtiva & $0,50 \pm 1,41$ & $0,00 \pm 0,00$ & $0,00 \pm 0,00$ & $0,00 \pm 0,00$ \\
\hline
\end{tabular}

Fonte: Dados da Pesquisa* Médias e Desvio padrão

${ }^{\star *} 0,045$ diferença estatisticamente significativa a um nível de significância de $5 \%(p \leq 0,05)$. 
Conforme o Gráfico 4 os itens corretos do MMSE do grupo suplementado tiveram um aumento na pontuação total ao longo do tempo de $55,8 \%$, já o grupo controle teve uma redução na pontuação total de 25,3\%. Realizando o teste não paramétrico para as medias (U-de Mann Whitney) obtemos significância estatística entre os grupos, ou seja, ao nível de $5 \%$ tivemos diferença entre as pontuações do teste cognitivo entre o grupo controle e o grupo suplementado.

Gráfico 04 - Comparativo do grupo controle $(n=4)$ e o suplementado $(n=4)$, segundo a soma de itens corretos no questionário cognitivo aplicados em quatro períodos distintos (0,30,60 e 90), Teresina, PI, 2018.

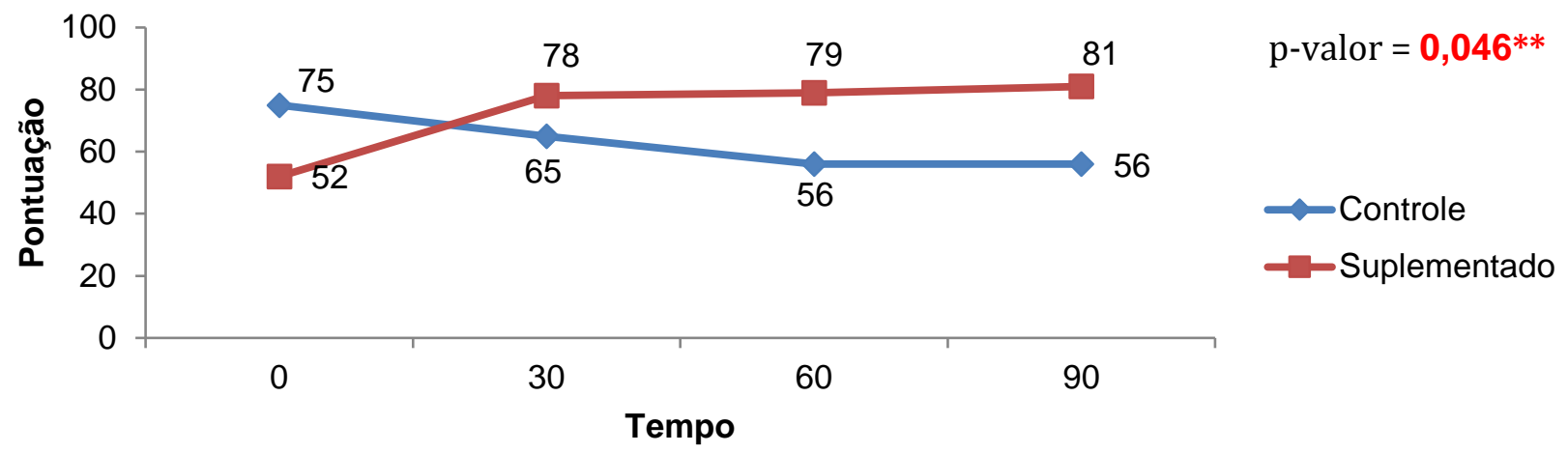

Fonte: dados da pesquisa, 2018.

${ }^{* *}$ diferença estatisticamente significativa a um nível de significância de $5 \%(p<0,05)$.

Verificou-se que todos os idosos com Alzheimer utilizavam as seguintes medicações: Hidroclorotiazida, Cloridrato de Donepezila, Hemifuramato Quetiapina, Sivastatina Hemifumarato de bisoprolol, Cloridrato de trazodona (LOREDON) com dosagens individualizadas. É sabido que os idosos utilizam outras medicações mas a escolha da suplementação como já relatado no objetivo, foi somente para avaliar efeitos da suplementação na função cognitiva e estado nutricional.

\section{DISCUSSÃO}

Como o estresse oxidativo tem sido implicado na progressão da DA, o estudo piloto foi desenvolvido para examinar os efeitos de $\omega-3$, magnésio e vitamina B12 em idosos com Alzheimer. Em comparação com controle, houve diferença significativa em 90 dias no mini teste mental, bem como houve evolução em relação ao risco para DCV. Assim, parece que a suplementação destes nutrientes pode exercer efeito de tratamento de Alzheimer, reduzindo o comprometimento cognitivo e funcional.

Há um estudo relatado na DA avaliando os efeitos da suplementação com combinação de antioxidantes nos biomarcadores do líquido cefalorraquidiano (LCR); medidas cognitivas e funcionais. Os indivíduos foram randomizados para um dos três grupos: 1) placebo, 2) combinação de antioxidantes (dose diária, $800 \mathrm{UI}$ de a-tocoferol, $500 \mathrm{mg}$ de vitamina C, $900 \mathrm{mg}$ de ácido alfa-lipóico), 3) coenzima Q10 (400 mg três vezes ao dia). (YURKO-MAURO K, et al., 2010). Embora o grupo de combinação antioxidante tenha mostrado uma redução nos níveis de F2-isoprostano no LCR, este grupo também exibiu declínio cognitivo mais rápido (MMSE) ao longo de 16 semanas do que o grupo placebo. Os autores concluíram que, embora os efeitos antioxidantes tenham sido alcançados com a dose específica e a combinação de antioxidantes, o rápido declínio nos escores do MEEM causou "cautela" e um ensaio clínico de longo prazo dessa combinação antioxidante foi justificado.

De acordo com os resultados desta pesquisa, os pacientes com DA apresentam distúrbios do estado nutricional, excesso de peso e obesidade, acúmulo de gordura visceral, que pode caracterizar uma resistência periférica a ação da insulina. Estudo realizado por Cova I, et al. (2017), demostram que pacientes com DA, apresentaram redução da massa tecidual e desidratação, aumento do componente de gordura em relação à massa muscular juntamente com declínio psicofuncional. 
Em um estudo realizado por Souza R, et al. (2013) com 131 idosos, os pesquisadores relacionaram a classificação de IMC com base em valores de referência da OMS com de Lipschitz, em que esses idosos receberam a classificação de sobrepeso, obesidade grau I e II da OMS para então comparar com as classificações de Lipschitz, sendo assim idosos com IMC acima de $27 \mathrm{~kg} / \mathrm{m}^{2}$ é classificado como sobrepeso. Portanto, $1,5 \%$ dos idosos foram classificados com baixo peso segundo a OMS, enquanto $48,1 \%$ foram classificados como eutróficos e 50,4\% sobrepeso, obesidade grau I e II. Agora para Lipschitz, relatou 16\% foram classifiacdos como baixo peso e $52,7 \%$ como eutróficos e $31,3 \%$ sobrepeso.

Santos, et al. (2013), relatou em uma experiência com idosos e demonstrou a ligação entre a obesidade central e presença de fatores de risco cardiovasculares, como HAS, resistência insulínica, DM tipo 2, hipertrigliceridemia e HDL baixo. E para Zhang X, et al. (2008) em um estudo realizado em Recife, observou que $1,9 \%$ das pessoas estão com baixo peso segudo a classificação da OMS e quando a classificação segue por Lipschitz, essa porcentagem passa a ser $12,3 \%$. Para o excesso de peso $47,2 \%$ da amostra e pela OMS foi de $65,1 \%$.

A CB reflete tanto a gordura subcutânea quanto a massa magra. Estudos sugerem que a CB tem alta correlação com o IMC e pode ser bom indicador em substituição ao IMC ou mais mensurador de avaliação do estado nutricional da população geriátrica (ARAUJO, FARIA, PEREIRA, 2007). Um estudo realizado por Garcia, Romani, Lira, 2007 com 308 idosos residentes em instituições asilares públicas e privadas em Pernambuco, constatou prevalência de $24,4 \%$ de desnutrição por meio da CB.

De fato, o estudo de melhoria da memória com ácido docosaexaenoico, ou MIDAS (Memory Improvement with Docosahexaenoic Acid Study / Estudo de melhoria da memória com ácido docosahexaenóico), um grande estudo aleatório e controlado com placebo, divulgado na revista Alzheimer's \& Dementia: The Journal of the Alzheimer's Association (Alzheimer \& demência: A revista da sociedade do Alzheimer), revelou que pessoas saudáveis com idade superior a 55 anos com queixas de memória que tomaram $900 \mathrm{mg}$ de cápsulas de DHA de algas durante seis meses duplicaram a percentagem de redução em erros em um teste para medição de desempenho de aprendizagem e memória comparado com aquelas que tomaram placebo, um benefício quase equivalente ao de ter habilidades de aprendizagem e memória de alguém três anos mais jovem.

Vale ressaltar que a vitamina B12 está associada a melhores escores gerais globais de cognição assim como, reduzem as condições de demência e Alzheimer elas também desempenham papeis importantes no ciclo da homocisteína, pois sem um suprimento adequado dessas vitaminas, a homocisteína se acumula intracelularmente causando demência dentre elas a DA (MIKKELSEN K, et al., 2016; MOORTHY D, et al., 2017).

Em um estudo realizado por EIBaz F, et al. (2016) constatou se que os pacientes com transtorno do déficit de atenção e hiperatividade, deficientes em magnésio tiveram escores mais baixos no WCST do que os nãodeficientes. A suplementação de magnésio melhorou as pontuações.

Estudos demonstraram os efeitos do magnésio na aprendizagem e na memória nos seres humanos. Níveis mais baixos de $\mathrm{Mg}$ no liquido cefalorraquidiano podem ser um fator promotor da degeneração neural e consequentemente declínio cognitivo uma vez que a deficiência desse mineral está associada com aumento do estresse oxidativo fator este que potencializa o surgimento da DA (ELBAZ F, et al., 2017 e VERONESE N, et al., 2015).

\section{CONCLUSÃO}

Sugere-se que a suplementação de $\omega-3$, magnésio e vitamina B12 em idosos com Alzheimer ocasionou melhoria significativa na função cognitiva no período de 90 dias (o grupo controle passou de uma $75 \%$ para $52 \%$ de acertos no teste enquanto que grupo suplementado de $52 \%$ para $81 \%$ acertos) e quanto ao estado nutricional houve alterações relevantes na média dos parâmetros antropométricos avaliados (peso $62,75 \mathrm{~kg} \mathrm{e}$ circunferência da cintura 99,25kg) dos idosos, após 60 dias quando comparado com período inicial $59,75 \mathrm{~kg}$ 
peso e 95,13 cm de circunferência da cintura. No entanto são necessárias mais pesquisas acerca desse tema para uma comprovação mais eficaz visto que não foi realizada análise quantitativa da dieta dos idosos, bem como a interação droga nutriente dos medicamentos utilizados por eles.

\section{REFERÊNCIAS}

1. ALLEN LH. How common is vitamin B-12 deficiency?. Am. J. Clin. Nutr.,2009; 89, 693S-696S.

2. ALZHEIMER'S ASSOCIATION. 2016 Alzheimer's disease facts and figures. Alzheimer Dement, 2016; 12(4):459-509.

3. ARAÚJO CR, et al. Análise do perfil nutricional de idosos do movimento da Terceira idade praticantes de hidroginástica. Revista Nutrir Gerais, 2007.

4. BIGUETI BCP, et al. Nutrientes essenciais na prevenção da doença de Alzheimer. Revista Ciências Nutricionais Online, 2018; v.2:18-25.

5. BLACKBURN GL, THORNTON PA. Nutritional assessment of the hospitalized patients. Medical Clinics North America, 1979; 63: 1103-1115.

6. BURR M L, PHILLIPS MK. Anthropomtric norms in the elderly. British Journal of Nutrition, 1984; 51:165-169.

7. CANHADA SL. A suplementação de ômega 3 na doença de Alzheimer: uma revisão sistemática. 2015. 43 p. Monografia (Bacharelado em Nutrição) - Universidade Federal do Rio Grande do Sul. Porto Alegre: UFRS, 2015.

8. CANSEV M, et al. Oral administration of circulating precursors for membrane phosphate des can promote the synthesis of new brain synapses. Alzheimer's \& Dementia 2008; 4: S153-68.

9. COMINETTI C, et al. Gênômica nutricional: dos fundamentos à nutrição molecular. Barueri, SP: Manole,2017.

10. CORREIA A, et al. Nutrição e doença de Alzheimer: programa nacional para a promoção da alimentação saudável direçãogeral da saúde. Lisboa, 2015.

11. COVA I, et al. Nutritional status and body composition by bioelectrical impedance vector analysis: A cross sectional study in mild cognitive impairment and Alzheimer's disease. PLoS One, 2017;12(2):e0171331.

12. COZZOLINO SMF. Bio disponibilidade de nutrientes. 3. ed. Barueri, SP: Manole, 2009.

13. ELBAZ F, ZAHRA S, HANAFY H. Magnesium, zinc and copper estimation in children with attention deficit hyperactivity disorder (ADHD). Egyptian Journal of Medical Human Genetics, 2017; v.18, n.2:153-163.

14. ELBAZ F, et al. Suplementação de magnésio em crianças com transtorno de déficit de atenção e hiperatividade. Egyptian Journal Medical Human Genetics, 2016; 17 (1): 63 - 70.

15. FRISANCHO AR. Antropometric Standards for the Assessment of Growth and Nutritional Status. University of Michigan, 1990; p. 189.

16. GARCIA ANM, et al. Indicadores antropométricos na avaliação nutricional de idosos: um estudo comparativo. Revista de Nutrição. Campinas, 2007; 20(4):371-378.

17. GREEN, R. \& Miller; J. W. Handbook of Vitamins 5th edn (edsZempleni, J. et al.) 447-489 (Taylor \& Francis, 2014). A comprehensive review of $\mathrm{B} 12$ biochemistry, nutrition and metabolism.

18. GUIGOZ Y, et al. The mini nutritional assessment; a practical assessment tool for grading the nutritional state of elderly patients. In: Guigoz, S. C., et al. The Mini Nutritional Assessment: MNA, Facts and Research in Gerontology. New York: Serdi, p. 15-59, 1999.

19. LIPSCHITZ DA, Screening for nutritional status in the elderly. Primare Care, $1994 ; 1: 55-67$.

20. MIKKELSEN K, et al. Cognitive decline: A vitamin B perspective. Maturitas, 2016; v. 93: 108-113.

21. MOLFINO A, et al. The role for dietary omega-3 fatty acids supplementation in older adults. Nutrients, 2014; 6(10):405873.

22. MOORTHY D, et al. Status of vitamins B-12 and B-6 but not of folate, homocysteine, and the methylenetetrahydrofolate reductase C677T polymorphism are associated with impaired cognition and depression in adults. J Nutr., 2012; 142(8):155460.

23. NATIONAL INSTITUTES OF HEALTH (NIH publication n. 00-4084). The practical guide identification, evaluation, and treatment of overweight and obesity in adults. Bethesda, MD: 2000.

24. SANTOS AA, et al. Sono, fragilidade e cognição: estudo multicêntrico com idosos brasileiros. Revista Brasileira de Enfermagem, 2013. vol. 66, n.3, pp.351-357.

25. SCHIEFERDECKER MEM, et al. Vitaminas, minerais e eletrólitos: aspectos fisiológicos, nutricionais e dietéticos. Rio de Janeiro: Rubio, 2016.

26. SOUZA R, et al. Avaliação Antropométrica em Idosos: Estimativas de peso e altura e concordância entre classificações de IMC. Revista Brasileira Geriatria e Gerontologia. Rio de Janeiro, 2013 vol.16 no.1.

27. VEDIN I, et al. Effectsofdha-rich n-3 fatty acid supplementation on gene expression in blood mononuclear leukocytes: the omega d study, 2012;7(4):e35425.

28. VERENOSE N, et al. Effect of oral magnesium supplementation on physical performance in healthy elderly women involved in a weekly exercise program: a randomized controlled trial. The American Journal of Clinical Nutrition, 2014; p 9;71(11):82632.

29. VOGIATZOGLOU A, et al. Vitamin B12 status and rate of brain volume loss in community-dwelling elderly. Neurology, 2008;71(11):826-32.

30. YURKO-MAURO K, et al. Beneficial effects of docosahexaenoic acid on cognition in age-related cognitive decline, 2010;6(6):456-64.

31. ZHANG X, et al. Prevalence and associated factors of overweight and obesity in a chinese rural population obesity. Obesity (Silver Spring), 2008; 16(1):168-71. 